\title{
A Layered Architecture for Fog Computing for an Effective Real Time Data Processing
}

\author{
Garima Joshi \\ Assistant Professor \\ Department of Computer \\ Science \& Engineering Shri \\ Vaishnav Institute of \\ Information \& Technology, \\ SVVV, Indore
}

\author{
Pooja Deshpande \\ Assistant Professor \\ Department of Computer \\ Science \& Engineering Shri \\ Vaishnav Institute of \\ Information \& Technology, \\ SVVV, Indore
}

\author{
Prasun Joshi \\ Research Scholar \\ IIIT, Banglore
}

\begin{abstract}
IoT (Internet of Things) is one of the greatest needs and requirement of the today's living. As we see we are in the era of sensor based devices and gadgets which are dealing with real time data with high volume that demands processing with high speed and velocity along with better security, not only the smart phones but smart homes, smart factory, smart grid, smart agricultural, even smart control systems like smart traffic A-Z are shifting towards IoT via sensors. When talking about the solution for processing and storing the large amount of data, one solution has come up is cloud but with increase in the application of sensors and real time devices, the resource management with high security of heterogeneous data is one of the key concern. We can say that cloud computing deals with computing and storage at the public cloud i.e. at data centers. Obviously, the user data takes longer duration to reach to the data centers and then it gets processed. So, fog computing is being introduced, that works at the user side i.e. at the private cloud thereby providing less time duration for processing the data with high security. It supports the virtualization and visualization and thus provides substantial amount of storage rather than trivial storage.
\end{abstract}

General Terms

IoT, private cloud, sensors

\section{Keywords}

Fog Nodes, Edge network

\section{INTRODUCTION}

Fog Computing is just as another cloud computing but at private cloud unlike cloud computing at public cloud. It provides scalability, pre-processing, profiling, monitoring, provisioning of resources, performance prediction, data management and security at private cloud. The various domains in today's life is moving towards the real time data processing from network connected objects. These objects may from different domain .Such objects performs actions and those actions can involve peer to peer communication or it may be human to machine communication For e.g. In gadgets like automatic doors, locks, human wearable sensor gadgets, camera and many more. Rapidly, this real time data processing can see in the domain such as manufacturing, smart transportation, mining and each public sector so thereby considering the data at extreme edge due to availability of thousands and millions of things across the globe, it should be analyze in efficient manner and in the time of milliseconds or even less.

\section{PROPERTIES OF FOG COMPUTING}

\subsection{Nearby To The End Users}

Unlike cloud computing ,Fog computing operates on private cloud so we can say that we can perform computing by staying more close to the User and hence to the sensors based devices. Being near to the user reduces the complexity of filtering the data and thereby reducing the real time data processing time.

\subsection{Location Awareness}

It is important that one must track the sensor devices at the user end to support mobility.as the nodes are able to infer its own area of placement as the fog node are associated with the geographical-distribution. So, location awareness is one of the major concern in the fog computing.

\subsection{Provides Resources For Latency Services Devices}

Servers at fog nodes enriched in resources for the sensor based objects. These objects may are network connected. Such objects are having properties of using the services of the network or sending the real time data over the network for the same it is mandatory required to perform computation on resources at the edge of the network.

\subsection{Supports Edge Analysis And Stream Mining}

IoT deals with the high volume of data .Not only that it also deals with different variety of data at high speed and with the high bandwidth.so to reduce the volume of real time data we can use various fog nodes that perform stream mining to cut down the delay period of computing and also it is helpful in analyze the data more appropriately.

\section{FUNCTIONS OF FOG COMPUTING}

\subsection{Monitoring}

Monitoring is one of the standard component in fog computing as it gives us all the relevant information for decision making and analysis like what is the work load at the particular instant of time, how much is the energy consumption and usage ratio of the nodes at the fog.

\subsection{Resource Management}

Resource Management deals with the allocation and deallocation of resources, interaction between resource to resource and resource to gadgets, which leads to efficient utilization of resources and thereby improving performance. As resources are placed in the distributed manner, 
communication between resources to resource is the major concern. So, it is required to maintain the resource pool. It is dynamic in nature, as the number of hosts and the requirements application changes with time.

\subsection{Profiling}

It builds and provides the information of resources and applications what is provided by monitors and knowledge discovery module.

\subsection{Knowledge Discovery And Stream Mining}

For the decision making if any kind of information is required by the resources or network then knowledge discovery plays important role as it store the historical data in the appropriate format. It is performed at the most upper layer of the layered architecture and as the data comes from the sensor based devices are in the continuous form stream mining is thus resultant in some patterns.

\subsection{Security}

Security is one of the major concerns in data and so in fog computing where the real time data gets processed. Authentication, Authorization, confidentiality and cryptography are the key responsibilities come under this

\subsection{Raw Data Management}

Data management includes the availability of data from one service to another service. It provides view of data comes from one service to give data to next services by simply perform query on the raw data.

\section{COMPONENTS OF FOG COMPUTING}

Fog Computing Layered architecture composed of four layers as shown in figure 1 with variant operations on different layers. Each layer has their own functions and all the independent in terms of their operability but is pipelined the terms of their processing. As each bottom layer's output is acting as an input for its corresponding top layer. These layers are responsible for performing profiling, provisioning, data management, data processing, stream mining, monitoring, security, prediction analysis of real time data comes from the multiple smart gadgets in various formats.

\subsection{Layer 1-Network Layer}

All the network edge resources are placed at this layer as this is the lower layer of the architecture. It is responsible for dealing directly with all types of sensors, gadgets, actuators those are used in various application as mentioned in the upcoming section Applications. By using API's this layer provides abstraction for communicating with the sensors and IoT devices via M2M protocols. Cisco-I Ox is the software which facilitates such type of abstractions.

\subsection{Layer 2-Management Layer}

It is responsible for the resource management, data management, monitoring and profiling. $\mathrm{DMo}$ (Data in Motion) is one of the tools by Cisco which supports such type of functions at this layer. Scheduling of resources includes allocation, de-allocation of resources for providing services. To maintain the availability of resources .To control flow of data from the resources. It calculates the overall computing times of individual fog-nodes and power consumption of the fog by continuous monitoring all the fog nodes at multiple locations.

\subsection{Layer 3-Security Layer}

After passing through bottom two layers the real time data is now required to be more authentic and authorized. Therefore the cryptographic algorithms and techniques are performed at this layer. The one of the tool that deals with security of real time data is Vortex. Vortex plays vital role in security thereby providing mobility for the real time data to be Ishared timely. As the fog architecture uses the same procedure, principles, protocols and controls and therefore it protects the fog nodes placed at heterogeneous locations Use the same physical security techniques. So, it is easy to determine the fraud at this layer of security.

\subsection{Layer 4 - Programming And Service Layer}

It is the most top layer of the architecture that provides abstraction from the functionality of the bottom 3 layers and performs the mining on the continuous data and thus provides knowledge and patterns for the various applications. For the mining of real time data the most important tools is ParStream. This layer provides the periodic data summaries and sends it to the cloud that performing the analysis on the IoT data.

\section{APPLICATION OF FOG COMPUTING}

\subsection{Climate Smart Agriculture}

Not only our country but whole world is the not remain untouched with this area of application .i.e. agriculture. As day by day technology grows the importance of smart equipment is the necessity of the today agriculture .From Soil Science measurement equipment, effect of climate on the food is the major issues in the today's era and without predicting the temperature or future climate positions we cannot even think sowing of cultivating and sowing seeds. So, IoT plays crucial role in smart agricultural. In Ecosystem climate and agriculture plays important role so FAO (Food and Agricultural Organization) of the United Nations introduced this term Climate Smart agriculture that increases the productivity and enhance adaptation by using the fog computing.

\subsection{Smart Traffic And Smart Vehicles}

In the case of emergency like passing the ambulance from the road side ways, all the vehicles nearby receives the signal by measuring the distance of both the ambulance and the corresponding vehicle and also speed of the nearby vehicle. Here, the traffic signals acts as a fog device. The communication between the vehicle and ambulance access points can be observed via Wi-Fi in such cases. Talking about the smart vehicles, connected cars are newly implemented concept in which using fog computing where connected cars ,traffic lights and access points behaves as the fog nodes and can interacts with each other safely.

\subsection{Smart Building}

Smart Building includes various sensors devices like smoke sensor for fire alarming or sensor for climate and temperature monitoring, sensors for smart parking, sensors for theft detection etc. It is not limited up-to the particular building but are associated with each and every room of that building .Each individual sensor device can act as a fog node and thus monitoring the real time data. It can also be considered that the smart gadgets and smart phones comes under smart building can become part of it. 


\subsection{Smart Grid}

Smart Grid is the most important as the power consumption of electricity increases with great amount. In the smart grid fog collector is responsible of collecting the data and controls the measurement process on itself. The filtering data is used as a report for Visualization.

\subsection{Self Operating Trains}

The sensors deployed at the ball bearing acts as the fog nodes and can sense the temperature and climate and report automatically to the train operator to avoid the accidents.

\subsection{Used In Survelllance}

Today each and every of us is the part of surveillance and why not it is very important not only to prevent the fraud but also to find the criminal .Fog nodes are placed in the Visual camera which monitors the real time data and detects anomalies. Visual camera that includes the fog nodes can also responsible for maintaining the privacy and confidentiality of images and do not reveals the contextual information to the unauthorized users.

\section{ADVANTAGES OF FOG COMPUTING}

\subsection{Decrease Network Congestion}

By placing fog nodes in between IoT devices and cloud and performing fog computing decreases the network congestion at cloud.

\subsection{Improves Quality Of Analysis}

As the variety of data comes into variant of formats the layered architecture of fog computing improves the quality of analysis and thus resultant decisions are better.

\subsection{Decrease Processing Latency}

Fog nodes present very close to the end user and perform processing so the work load on the nodes is according to the location of them. Thus, it decreases the processing latency.

\subsection{Avoids Resource Contention}

Avoiding Resource contention is one the big challenge in cloud which is resolve in Fog computing by providing resource management.

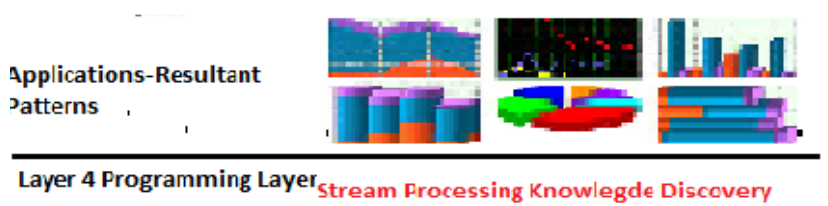

Layer 3 Security Layer Authentication,Authorization,Confidentiality

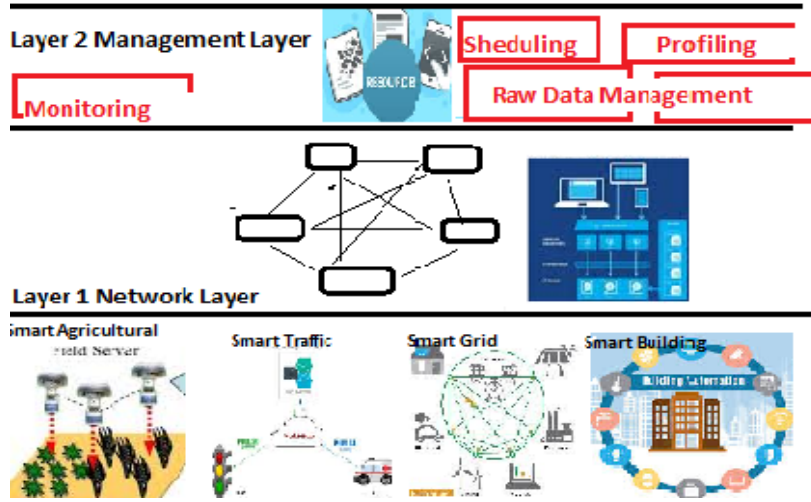

Figure 1: A Layered Architecture for effective fog computing

\section{CONCLUSION}

In the Paper we have introduced a layered architecture that is suitable to implement various operations by abstracting each operation with another .I have suggested various suitable tools at each stage for implementing the operation. Future work includes implementation of this architecture by using tools for performing such operation at different layer and thus will result in providing better results of Fog computing.

\section{REFERENCES}

[1] Fog Computing and the Internet of Things" Extend the cloud to where the things are?", Cisco, 2015.

[2] S. Sanjeev, S. Thus, R.V. College of Engineering, Bengaluru, "A Survey of Fog Computing and Its Applications" IJARIIT, Volume3, Issue2, 2017

[3] S. Yi, Zijiang Hao, Zhengrui Qin, and Qun Li“Fog Computing: Platform and Applications", IEEE 2015

[4] Amir Vahid Dastjerdi and Rajkumar Buyya, University of Melbourne "Fog Computing: Helping the Internet of Things Realize its Potential", IEEE computer society, 2016

[5] Amir Vahid Dastjerdi, Harshit Gupta, Rodrigo N Calheiros, Soumya K. Ghosh, and Rajkumar Buyya, "Fog Computing Principles, Architectures, and Applications",2016

[6] Salvatore J. Stolfo, Malek Ben Salem, Angelos D Keromytis Fog Computing: Mitigating Insider Data Theft Attacks in the Cloud, IEEE, 2012

[7] Ivan Stojmenovic, Sheng Wen" The Fog Computing Paradigm: Scenarios and Security Issues" Federated Conference on Computer Science and Information Systems, 2014

[8] Shanhe Yi, Cheng Li, Qun Li A Survey of Fog Computing: Concepts, Applications and Issues, ACM, 2015. 